\title{
Caractéristiques de la résistance du cultivar de pêcher Rubira à Myzus persicae Sulzer
}

\author{
L Kfoury, G Massonié* \\ INRA, unité de recherches de zoologie, BP 81, F33883 Villenave-d'Ornon cedex, France
}

(Reçu le 23 septembre 1994 ; accepté le 10 juillet 1995)

\begin{abstract}
Résumé - D'après les résultats obtenus, la résistance du cultivar de pêcher Rubira aux fondatrigènes aptères de Myzus persicae est induite et systémique. Cette résistance est mise en place en réponse aux piqûres alimentaires du puceron. La phase initiale ou période d'initiation est suivie d'une période de latence pendant laquelle la présence de l'insecte n'est plus nécessaire. La résistance est observée au terme d'une période d'initiation de $2 \mathrm{~h}$, suivie d'une période de latence de $18 \mathrm{~h}$. Le retrait des pucerons provoque l'affaiblissement progressif puis la disparition de la résistance dans les $48 \mathrm{~h}$. L'apparition de la résistance précède celle des premières réactions (décolorations puis réactions nécrotiques localisées) des tissus végétaux aux piqûres de l'insecte. La discussion concerne d'une part les relations entre la résistance et les réactions nécrotiques, d'autre part la comparaison des résistancs induites par le puceron et par des acariens.
\end{abstract}

pêcher / puceron / résistance / nécroses / hypersensibilité

Summary - Characteristics of the resistance of the peach cultivar Rubira to Myzus persicae Sulzer. This work relies upon the comparison of the timing of aphid behaviour when young plants are infested for the first or second time by the fundatrigeniae apterae of Myzus persicae. The mechanisms of plant resistance are initiated by the feeding punctures and evolve even in the absence of aphids. The initiation stage is short, around $2 h$, and followed by latency, around $18 \mathrm{~h}$. After aphid removal the systemic acquired resistance decreases and disappears in the following $48 \mathrm{~h}$. The systemic acquired resistance appears before the emergence of local discolourations and necrotic areas restricted to the aphid feeding punctures. Mechanical wounding failed to initiate the systemic acquired resistance. The results are discussed on the basis of the present knowledge of induced resistances and hypersensitivity to arthropods with piercing mouthparts, mites and aphids.

peach / aphid / resistance / necrosis / hypersensitivity

\section{INTRODUCTION}

L'étude de la résistance des plantes à leurs parasites permet de distinguer 2 types de résistance: la résistance constitutive et la résistance induite. Lorsque la résistance est constitutive, les fac- teurs de résistance sont présents avant l'infestation ; lorsqu'elle est induite, leur apparition est provoquée par l'infestation.

La résistance induite implique l'activation des gènes de défense de la plante en réponse à l'infestation par des champignons (Collinge et

\footnotetext{
* Correspondance et tirés à part
} 
Slusarenko, 1987), des bactéries (Lamb et al, 1989), des virus (Fritig et al, 1987), des acariens (Bronner et al, 1991). La première étape de la mise en place de la résistance induite consiste en la reconnaissance du parasite par la plante. La reconnaissance est suivie de l'apparition de signaux qui déclenchent la résistance dans les tissus voisins et éloignés si la résistance est systémique. Les signaux ou éliciteurs peuvent avoir 2 origines: les exoéliciteurs sont directement injectés dans la plante par le parasite, les endoéliciteurs sont synthétisés par la plante en réponse à divers agents inducteurs, biotiques ou abiotiques. Plusieurs types de résistances induites ont été signalés (Ryals et al, 1994). Ces résistances peuvent être associées ou non à des réactions nécrotiques (Jakobek et Lindgren, 1993). L'hypersensibilité est caractérisée par l'apparition de nécroses localisées aux points d'infestation. Ces nécroses témoignent de l'induction des gènes de défense des cellules vivantes voisines des cellules mortes (Collinge et Slusarenko, 1987).

Chez les arthropodes piqueurs, le caractère induit de certaines résistances est bien établi avec des acariens, au moyen d'un test biologique consistant à comparer l'influence sur l'acarien d'une plante jusque-là non infestée à celle d'uneplante préalablement infestée. La résistance induite n'est pas toujours associée à des nécroses (Karban et Myers, 1989). L'une d'entre elles est du type hypersensible (Bronner et al, 1991). Elle confère par ailleurs une plus grande sensibilité à une autre espèce d'acariens (Westphal et al, 1992).

Peu de travaux ont été consacrés à la mise en évidence du caractère induit des résistances intervenant contre des pucerons (Kogan et Paxton, 1983). Pourtant ces insectes provoquent fréquemment des réactions induites, notamment des déformations du feuillage et des réactions nécrotiques (Miles, 1989) observées parfois sur les plantes sensibles (Dorschner et al, 1987). L'infestation peut modifier la translocation de la sève élaborée et sa disponibilité pour les pucerons (Way et Cammell, 1970). Elle peut provoquer une augmentation (Niemeyer et al, 1989 ; Leszczynski et Dixon, 1990) ou une diminution (Miles, 1990) de la teneur en substances phénoliques des tissus foliaires. Elle peut provoquer l'activation de gènes de défense de la plante (Van der Westhuizen et Botha, 1993). Les blessures mécaniques infligées par les pucerons aux tissus végétaux et l'action de leurs enzymes salivaires pourraient provoquer l'apparition de substances volatiles (Hildebrand et al, 1993) et d'oli- gogalacturonides (Campbell et Dreyer, 1990) susceptibles d'intervenir comme éliciteurs.

$M$ persicae, puceron vert du pêcher, est un puceron dioécique dont l'hôte primaire principal est le pêcher (Hille Ris Lambers, 1946). II est à l'origine de nombreux dégâts particulièrement par son rôle vecteur de virus phytopathogènes (Sharka). Nous avons montré que le cultivar Rubira présentait une résistance à ce puceron, résistance associée à des réactions nécrotiques (Massonié et Maison, 1979). Diverses données de la littérature révèlent que certaines variétés de pêcher peuvent développer des résistances induites contre des champignons parasites (Braun et Helton, 1971 ; Biggs et Peterson, 1990). Par ailleurs, Mclntyre et al (1981) signalent que les virginogènes de $M$ persicae peuvent être affectés par une résistance systémique (résistance du tabac au virus de la mosaïque du tabac). Compte tenu de ces données, l'objectif du travail présenté concerne la caractérisation (induction et systémie) de la résistance de Rubira à $M$ persicae et sa liaison avec les réactions nécrotiques du végétal.

\section{MATÉRIELS ET MÉTHODES}

\section{Matériel végétal}

Les cultivars de pêcher GF 305 et Rubira clone $\$ .2605$ sont des lignées pures. Ils sont utilisés au stade jeune plant issu de semis, le premier pour l'élevage de l'insecte, le second pour l'étude de la résistance. Le comportement des semis par rapport à $M$ persicae est identique à celui des arbres (Massonié et al, 1982). Les semis des 2 cultivars sont cultivés dans une serre dont la température est régulée entre $18^{\circ} \mathrm{C}$ et $28^{\circ} \mathrm{C}$. les jeunes plants sont infestés lorsque la hauteur de leur tige est comprise entre 30 et $50 \mathrm{~cm}$. Avant la mise en place des essais tous les rameaux axillaires sont éliminés, à l'exception d'une série expérimentale impliquant la présence d'un rameau axillaire basal.

\section{Matériel animal}

Un élevage de masse de $M$ persicae a été constitué à partir d'une fondatrice prélevée en verger de pêcher. Cet élevage est maintenu depuis 3 ans, sur de jeunes plants du cultivar sensible GF305, dans une pièce climatisée aux conditions suivantes : température $20^{\circ} \mathrm{C} \pm$ $1^{\circ} \mathrm{C}, 16 \mathrm{~h}$ d'une lumière artificielle d'intensité d'environ $30 \mu$ moles photons $\mathrm{m}^{-2} \mathrm{~s}^{-1}$, hygrométrie comprise entre 70 et $90 \%$. Chaque plant reçoit 5 fondatrigènes aptères adultes. Les premiers adultes de la génération suivante apparaissent de 10 à 12 j plus tard. Ils assu- 
rent le maintien de l'élevage de masse. Les adultes aptères ayant moins de $1 \mathrm{sem}$ de vie imaginale et les larves du premier stade âgées de moins de $24 \mathrm{~h}$ sont utilisées pour la réalisation des essais.

\section{Protocole des essais}

Dans les essais 1 à 6 , l'observation effectuée consiste en la mesure de la résistance de chaque plant en fonction de différents traitements. Ces essais se décomposent donc en 3 opérations successives : le traitement, le délai entre la fin du traitement et le début du test de mesure de la résistance, le test de mesure. Ce dernier consiste au dépôt de 10 pucerons aptères adultes dénommés indicateurs sur l'apex de chaque plant (10 plants par traitement). La résistance est alors évaluée par le dénombrement des pucerons indicateurs qui restent sur les plants en fonction du temps. Pour chaque essai et pour chaque dénombrement, les traitements sont comparés, à l'aide des tests non paramétriques de Mann-Whitney ou de Kruskal-Wallis. Lorsque le test de Kruskal-Wallis conclut à une différence globale, les moyennes sont comparées 2 à 2 , au niveau $5 \%$, par la méthode de la plus petite différence significative (Scherrer, 1984). Les résultats des dénombrements sont définis par leur moyenne et leur intervalle de confiance $(P=0,05)$. Leur présentation est limitée à celle du dénombrement caractérisé par l'apparition de la première différence significative entre la moyenne du témoin de l'essai et de l'un des traitements. Le temps $t_{1}$ écoulé (en $\mathrm{h}$ ) entre l'infestation initiale $\left(T_{0}\right)$ et ce dénombrement particulier $\left(T_{1}\right),\left(t_{1}=T_{1}\right.$ $-T_{0}$ ), est indiqué.

Le traitement correspond soit à une infestation par des pucerons, soit à des blessures mécaniques, soit à l'absence de toute intervention (témoin) (tableau I). Les pucerons utilisés lors des traitements sont des larves du premier stade ou des adultes aptères. Ces derniers peuvent être libres ou encagés. Les pucerons libres, leurs larves et leur miellat sont éliminés en fin de traitement par lavage à l'eau du robinet. Les plants témoins sont également lavés. Les cagettes, transparentes et aérées, sont disposées sur des parties de la tige peu lignifiées pour que les pucerons acceptent de s'alimenter. La distance maximale entre les cagettes et l'apex des plantes est d'environ $7 \mathrm{~cm}$ lorsque les cagettes sont en position sous-apicale, position dénommée Ta, d'environ $50 \mathrm{~cm}$ lorsqu'elles sont sur un rameau basal, position dénommée $\mathrm{Rb}$. Les pucerons encagés demeurent en place pendant le test car ils ne peuvent être confondus avec les pucerons libres et leur présence renforce l'expression de la résistance (cf Durée d'expression). Les plants témoins portent également des cagettes vides. Les blessures mécaniques consistent en une excision annulaire à l'emplacement de la cagette placée sur la tige apicale. Les excisions ont environ $7 \mathrm{~cm}$ de hauteur. Elles sont profondes ou superficielles. Dans le premier cas (EP), elles éliminent le phloème, dans le second (ES), elles atteignent tout au plus le parenchyme cortical. Les blessures ont été effectuées $24 \mathrm{~h}$ avant le test.
Dans l'essai 7, l'observation effectuée concerne les délais d'apparition des dégâts sur les plants. Les dégâts sont classés par ordre de sévérité croissante : i) décolorations discrètes du feuillage, ii) stigmonoses ou petites tâches nécrotiques entourées par un halo rouge et localisées autour du point de piqûre, iii) dessèchements foliaires locaux, iv) flétrissement de feuilles apicales, v) feuilles à l'aspect en faucille par suite des modifications introduites dans leur croissance. Le protocole utilisé ne prend pas en considération le comportement de piqûre des pucerons et ses conséquences éventuelles sur les délais d'apparition des dégâts. Les résultats présentés concernent le délai minimum d'apparition des dégâts ou délai compris entre le dépôt des pucerons et la première observation des dégâts. Cette valeur est utilisée pour préciser si l'apparition des dégâts précède ou non celle de la résistance induite.

Le détail des traitements de l'essai 7 est présenté dans le tableau I. Ils consistent en une infestation limitée dans le temps par des pucerons libres ou encagés en position sous-apicale (10 plants par traitement). L'apparition des dégâts sur les plants est suivie après le retrait des cagettes et l'élimination des pucerons par lavage. Le traitement 5 est caractérisé par une double infestation : pendant $48 \mathrm{~h}$ par 20 pucerons encagés puis pendant $8 \mathrm{~h}$ (de 40 à $48 \mathrm{~h}$ ), par 10 pucerons libres déposés sur l'apex des plantes.

Les essais ont été réalisés, contrairement à l'élevage des pucerons et la culture des plants, dans une serre dépourvue d'un système de refroidissement efficace. Ils ont été effectués à différentes époques de l'année, donc dans des conditions d'environnement très différentes. Lorsque la température extérieure est élevée celle de la serre peut fluctuer sur $24 \mathrm{~h}$ entre 20 et $45^{\circ} \mathrm{C}$ et demeurer supérieure à $30^{\circ} \mathrm{C}$ pendant près de $6 \mathrm{~h}$.

\section{RÉSULTATS}

\section{Résistance induite}

\section{Mise en évidence}

Les différents traitements d'infestation par les pucerons (tableau I, essais 1 et 2) provoquent, dans des délais plus brefs que les témoins, le départ des pucerons indicateurs (tableau II, essais 1 et 2). Quand le délai entre le traitement, de durée limitée à $6 \mathrm{~h}$, et le test de mesure est nul, la valeur de $t_{1}$ est de $20 \mathrm{~h}$; quand ce délai est de $40 \mathrm{~h}$, la valeur de $t_{1}$ est de $3 \mathrm{~h}$.

Ces résultats mettent en évidence l'influence du traitement et du délai entre celui-ci et le test de mesure. L'induction de la résistance de Rubira est donc caractérisée par une période d'initiation et par une période de latence. La pre- 
Tableau I. Caractéristiques des essais concernant l'étude des relations entre le cultivar de pêcher Rubira et $M$ persicae.

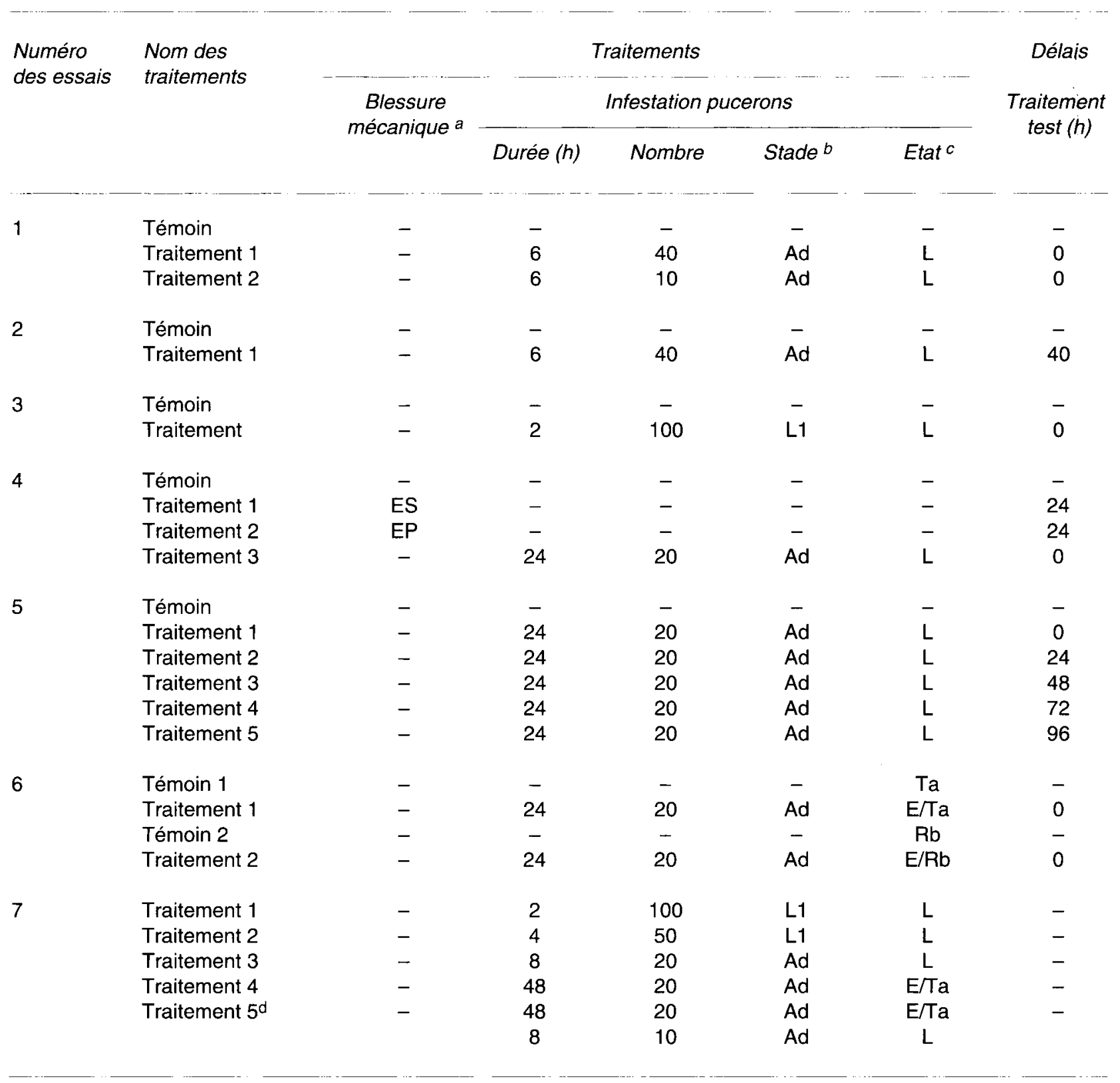

\footnotetext{
a Les blessures mécaniques sont notées ES (excision superficielle) ou EP (excision profonde) ; ${ }^{b} \mathrm{Ad}=$ adulte, $L 1=$ larve du $1^{\mathrm{er}}$ stade ; c les pucerons sont soit libres (L) ou encagés $(E)$. La cage est soit placée sur la tige apicale (Ta) ou le rameau basal (Rb). Lors de l'essai 6, les plants témoins portent une cage vide ; ${ }^{d}$ les pucerons libres sont déposés $40 \mathrm{~h}$ après les pucerons encagés.
}

mière correspond au déclenchement par les pucerons du ou des mécanismes de résistance, la seconde à l'évolution de ce(s) mécanisme(s) en l'absence des pucerons.

\section{Induction}

Les traitements par infestation avec soit 10 ou 40 adultes soit 100 larves (tableau I, essais 1 et 3 ) provoquent, dans des délais plus brefs que les témoins, le départ des pucerons indicateurs (tableau II, essais 1 et 3 ). Les traitements par des blessures mécaniques, superficielles ou profondes (tableau I, essai 4), provoquent le départ des pucerons indicateurs dans les mêmes délais que le témoin (tableau II, essai 4). Ces délais sont significativement supérieurs à ceux observés avec le traitement d'infestation par les pucerons. 
Tableau II. Influence des traitements et du délai entre le traitement et le test (tableau I) sur les effectifs de $M$ persicae à l'instant $t_{1}$.

\begin{tabular}{|c|c|c|c|}
\hline Essais & Traitements & $\mathrm{t}_{1}^{\mathrm{a}}(h)$ & Effectifs b \\
\hline 1 & $\begin{array}{c}\text { Témoin } \\
\text { Traitement } 1 \\
\text { Traitement } 2\end{array}$ & 20 & $\begin{array}{l}8,7 \pm 1,6^{a} \\
7,0 \pm 1,2^{b} \\
6,5 \pm 1,7^{b}\end{array}$ \\
\hline 2 & $\begin{array}{c}\text { Témoin } \\
\text { Traitement } 1\end{array}$ & 3 & $\begin{array}{l}9,3 \pm 1,1^{a} \\
6,4 \pm 1,2^{b}\end{array}$ \\
\hline 3 & $\begin{array}{c}\text { Témoin } \\
\text { Traitement } 1\end{array}$ & 18 & $\begin{array}{l}9,7 \pm 1,0^{a} \\
7,4 \pm 1,3^{b}\end{array}$ \\
\hline 4 & $\begin{array}{c}\text { Témoin } \\
\text { Traitement } 1 \\
\text { Traitement } 2 \\
\text { Traitement } 3\end{array}$ & 12 & $\begin{array}{l}7,3 \pm 1,3^{a} \\
7,8 \pm 2,3^{a} \\
6,2 \pm 1,9^{a} \\
2,0 \pm 1,7^{b}\end{array}$ \\
\hline 5 & $\begin{array}{c}\text { Témoin } \\
\text { Traitement } 1 \\
\text { Traitement } 2 \\
\text { Traitement } 3 \\
\text { Traitement } 4 \\
\text { Traitement } 5\end{array}$ & 15 & $\begin{array}{l}7,9 \pm 0,8^{a} \\
1,4 \pm 1,7^{b} \\
3,8 \pm 2,3^{a b} \\
5,5 \pm 1,9^{a} \\
6,4 \pm 1,2^{a} \\
7,4 \pm 1,4^{a}\end{array}$ \\
\hline 6 & $\begin{array}{c}\text { Témoin } 1 \\
\text { Traitement } 1 \\
\text { Témoin } 2 \\
\text { Traitement } 2\end{array}$ & 14 & $\begin{array}{l}6,7 \pm 0,9^{a} \\
3,0 \pm 1,1^{b} \\
6,8 \pm 1,8^{a} \\
3,5 \pm 1,8^{b}\end{array}$ \\
\hline
\end{tabular}

a $t_{1}=\left(T_{1}-T_{0}\right)$ avec $T_{0}$ : moment de l'infestation des plants par les pucerons indicateurs et $T_{1}:$ moment de l'apparition de la première différence significative entre la moyenne du témoin de l'essai et de l'un des traitements ; b moyennes \pm intervalles de confiance $(P=$ $0,05)$. Les moyennes de chaque essai suivies de lettres différentes sont significativement différentes $(P<0,05)$.

Les larves et les adultes aptères de $M$ persicae induisent la résistance de Rubira. L'induction intervient lors d'une infestation de durée limitée à $2 \mathrm{~h}$. L'influence de périodes d'infestation plus courtes n'a pas été étudiée. Dans nos conditions expérimentales, contrairement à l'infestation par puceron, les blessures mécaniques n'ont pas déclenché la résistance induite de Rubira.

\section{Durée d'expression}

En fonction de la durée du délai entre le traitement par infestation pendant $24 \mathrm{~h}$ avec des pucerons adultes et le test de mesure de la résistance (tableau I, essai 5), nous observons les faits suivants (tableau II, essai 5 ) :

- i) pour les durées inférieures à $48 \mathrm{~h}$, les plants ayant subi des traitements sont délaissés par les pucerons indicateurs avant le témoin ;
- ii) pour les durées comprises entre $48 \mathrm{~h}$ et $96 \mathrm{~h}$, ils sont délaissés par les pucerons indicateurs dans les mêmes délais que le témoin.

La résistance induite de Rubira s'atténue après le retrait des pucerons initiateurs et disparaît $48 \mathrm{~h}$ après le retrait de ces derniers.

\section{Systémie}

Les traitements par des pucerons encagés soit sur la tige apicale soit sur le rameau basal (tableau I, essai 6) provoquent tous deux, dans des délais inférieurs à ceux des témoins, le départ des pucerons indicateurs (tableau II, essai 6).

La résistance induite de Rubira est systémique. Dans nos conditions expérimentales, cette systémie agit au moins sur $50 \mathrm{~cm}$, distance moyenne entre les cagettes placées sur le rameau basal et l'apex des plants. 


\section{Délai d'apparition des dégâts}

Les dégâts et leur délai minimum d'apparition, sont indiqués au tableau III. L'apparition des dégâts d'un type donné peut s'échelonner sur $12 \mathrm{~h}$. Après une infestation de $2 \mathrm{~h}$, les dégâts provoqués par les larves demeurent limités à des décolorations et à des stigmonoses. Après une infestation de $4 \mathrm{~h}$, les larves provoquent l'ensemble des dégâts décrits. L'apparition des décolorations et stigmonoses est plus précoce après une infestation de $4 \mathrm{~h}$. La précocité d'apparition des stigmonoses et des dessèchements foliaires est plus grande après infestation par les adultes mais ces derniers ont infesté les plants plus longtemps que les larves $(8 \mathrm{~h})$. Les stigmonoses demeurent distinctes bien qu'elles puissent être rapprochées, contiguës mais non confluantes. Les délais d'apparition des flétrissements foliaires et des feuilles en faucille sont peu affectés par le stade de développement et la durée de séjour des pucerons.

Les pucerons encagés (tableau I, essai 7, traitement 4) ne provoquent que des stigmonoses localisées sur la portion de tige dont ils disposent. Les pucerons libres déposés sur des plants $40 \mathrm{~h}$ après le début de l'infestation par des pucerons encagés (tableau I, essai 7 , traitement 5 ) provoquent l'ensemble des dégâts décrits au tableau III.

La comparaison des délais d'apparition des dégâts provoqués par $M$ persicae et de la résistance induite montre que cette dernière précède généralement de quelques heures l'apparition des premières réactions visibles de Rubira, mais le décalage peut être plus important. Ainsi l'infestation pendant $2 \mathrm{~h}$ avec 100 larves du premier stade provoque l'apparition de la résistance induite $18 \mathrm{~h}$ après le retrait des larves alors que les premières décolorations du feuillage n'apparaissent que $56 \mathrm{~h}$ après le retrait des larves.

\section{DISCUSSION ET CONCLUSION}

L'infestation de jeunes plants du cultivar de pêcher Rubira par les fondatrigènes aptères adultes de $M$ persicae ou par leurs larves du premier stade a pour conséquence de provoquer des réactions induites caractérisées par l'apparition d'une résistance systémique et de dégâts.

La résistance systémique n'est observée qu'au terme d'un délai de mise en place. Celle-ci est déclenchée par les piqûres du puceron (phase d'initiation) puis elle se développe en l'absence de l'insecte pendant une période de latence. La durée de la phase d'initiation est brève car une infestation de $2 \mathrm{~h}$ est efficace. La durée de la période de latence est d'environ $18 \mathrm{~h}$. La présence du puceron s'avère indispensable pour assurer le maintien de la résistance systémique sinon elle s'affaiblit et disparaît en moins de 48 h, au terme de la période d'expression de la résistance.

Dans nos conditions expérimentales, contrairement à l'infestation par les pucerons, les blessures mécaniques des tissus végétaux n'ont induit ni une résistance de Rubira aux pucerons, ni l'apparition de dégâts de type nécrotique. Ceux-ci, révélés par des décolorations et des stigmonoses, sont le résultat de la mort de cellules et de modifications biochimiques des cellules vivantes situées au voisinage des cellules mortes. La résistance induite paraît donc être corrélée à des perturbations dans le fonctionnement des cellules végétales introduites par l'infestation. L'observation visuelle des dégâts nécrotiques est précédée parfois de plusieurs heures par l'expression de la résistance. La liai-

Tableau III. Délais minimum (en heures) d'apparition des dégâts sur les plants de Rubira en fonction des modalités d'infestation (tableau I, essai 7) par M persicae.

Dégâts

-

Décolorations foliaires

Stigmonoses

72

Dessèchements foliaires

Flétrissements foliaires

et feuilles en faucille
Traitement 1

Traitement 2

Traitement 3

\section{0}

45

45

72 
son éventuelle entre la résistance induite et les dégâts interviendrait donc avant l'apparition de ces derniers.

Les observations sont différentes de celles rapportées dans d'autres cas de résistance induite des plantes à des arthropodes. Ainsi, la résistance de Solanum dulcamara L à Aceria cladophtirus Nalepa est induite par cet acarien dans un délai comparable à celui que nous observons avec le modèle Rubira/M persicae mais elle présente une durée d'expression d'au moins $40 \mathrm{j}$ (Westphal et al, 1991). Cette résistance est associée à une réaction hypersensible caractérisée par la synthèse de protéines nouvelles et visualisée par l'apparition de nécroses localisées aux points de piqûre (Bronner et al, 1991). Il a été également montré que la résistance induite intervenant contre une autre espèce d'acariens pouvait être provoquée par des blessures mécaniques expérimentales (Karban, 1985).

Les différences du comportement alimentaire entre les pucerons et les acariens peuvent être invoquées pour expliquer ces observations. Les pucerons s'alimentent en prélevant la sève élaborée et la pénétration de leurs stylets jusqu'au phloème ne provoque que rarement la mort des cellules végétales. Au contraire, les acariens vident les cellules de leur contenu, ce qui provoque leur mort. Comme l'apparition des premiers signaux intervenant dans l'initiation des résistances induites est probablement associée à la mort des cellules végétales (Collinge et Slusarenko, 1987), les différences de comportement alimentaire pourraient avoir des répercussions importantes sur la quantité d'éliciteur libéré et sur l'intensité de la résistance induite (Dean et Kuc, 1985).

Si des réactions nécrotiques localisées sont associées aux résistances de type hypersensibilité, celles observées sur le pêcher Rubira en liaison avec la résistance aux pucerons ne sont pas obligatoirement les témoins d'une telle réaction. Cette résistance induite pourrait être par exemple initiée par la dégradation de substances constitutives en réponse à l'action conjuguée des blessures mécaniques et des enzymes salivaires des pucerons. Cette dégradation libérerait des substances biocides, responsables de la mort des cellules (Miles, 1990) et les réactions nécrotiques ne seraient alors que des conséquences secondaires de la résistance.

Pour comprendre les mécanismes de la résistance systémique de Rubira à $M$ persicae, il faudra développer des recherches dans 3 voies : i) dans quels tissus végétaux intervient la résistan- ce, ii) quels sont les allélochimiques impliqués dans cette résistance, iii) quels sont les moyens d'action du puceron. Les techniques actographiques d'étude du comportement alimentaire devraient permettre d'apporter un début de réponse à la première question (Massonié, 1986). La deuxième question est difficile à aborder car l'intervention des allélochimiques est précoce et se situe dans un ensemble complexe de réactions induites (Miles, 1990 ; Campbell et Dreyer, 1990). La réponse à la troisième question implique une étude préalable concernant le rôle d'autres pucerons dans la mise en place de la résistance décrite, afin de pouvoir réaliser une approche comparative.

En conclusion, les résultats obtenus soulignent la complexité des interactions plante-puceron et les difficultés de leur interprétation. L'intérêt de l'interaction présentée est de sortir de la discrétion habituelle des interactions plante-puceron associées aux piqûres alimentaires, discrétion qui rend leur étude difficile : «An interaction, the net result of which is an apparent absence of effects, is inherently difficult to investigate» (Miles, 1990).

\section{REMERCIEMENTS}

Les auteurs remercient les lecteurs du manuscrit : $\mathrm{P}$ Anglade et les lecteurs anonymes de la revue agronomie. La participation technique de $\mathrm{P}$ Maison a été fort utile et nous l'en remercions vivement.

\section{RÉFÉRENCES}

Biggs AR, Peterson CA (1990) Effect of chemical applications to peach bark wounds on accumulation of lignin and suberin and susceptibility to Leucostoma persoonii. Phytopathol 80, 9, 861-865

Braun JW, Helton AW (1971) Induced resistance to Cytospora in Prunus persica. Phytopathol 61, 685687

Bronner R, Westphal E, Dreger F (1991) Pathogenesis related proteins in Solanum dulcamara $L$ resistant to the gall mite Aceria caldophthirus (Nalepa) (syn Eriophyes cladophthirus Nal). Physiol Mol Plant Pathol 38, 93-104

Campbell BC, Dreyer DL (1990) The role of plant matrix polysaccharides in aphid plant interactions. In : Aphid Plant Genotype Interactions (RK Campbell, RD Eikenbary, eds), Elsevier, Amsterdam, 149-170

Collinge DB, Slusarenko AJ (1987) Plant gene expression in response to pathogens. Plant $\mathrm{Mol} B \mathrm{Biol} 9$, 389-410 
Dean RA, Kuc J (1985) Induced systemic protection in plants. Trends Biotechnol 3, 125-129

Dorschner KW, Ryan JD, Johnson RC, Eikenbary RD (1987) Modification of host nitrogen levels by the greenbug (Homoptera: Aphididae): its role in resistance of winter wheat to aphids. Environ Entomol $16,34,1007-1011$

Fritig B, Kauffman S, Dumas B, Geoffroy P, Kopp M, Legrand $M$ (1987) Mechanism of the hypersensitivity reaction of plants. In : Plant Resistance to Viruses, Wiley, Chichester (Ciba Foundation Symposium), 133, 92-108

Hildebrand DF, Brown GC, Jackson DM, Hamilton Kemp TR (1993) Effects of some leaf emitted volatile compounds on aphid population increase. J Chem Ecol 19, 1875-1887

Hille Ris Lambers D (1946) The hibernation of Myzus persicae Sulzer and some related species, including a new one (Hemip Aphididae). Bul Entomol Res 37, 197-199

Jakobek JL, Lindgren PB (1993) Generalized induction of defense responses in bean is not correlated with the induction of the hypersensitive reaction. Plant Cell 5, 49-56

Karban R (1985) Resistance against spider mites in cotton induced by mechanical abrasion. Entomol Exp App/37, 137-141

Karban R, Myers JH (1989) Induced plant responses to herbivory. Ann Rev Ecol Syst 20, 331-348

Kogan M, Paxton J (1983) Natural inducers of plant resistance to insects. Am Chem Soc 153-171

Lamb CJ, Lawton MA, Dron M, Dixon RA (1989) Signals and transduction mechanisms for activation of plant defenses against microbial attack. Cell 56, 215-224

Leszczynski B, Dixon FG (1990) Resistance of cereals to aphids: interaction between hydroxamic acids and the aphid Sitobion avenae (Homoptera: Aphididae). Ann Appl Biol 117, 21-30

Mc Intyre JL, Dodds JA, Hare JD (1981) Effects of localized infections of Nicotiana tabacum by tobacco mosaic virus on systemic resistance against diverse pathogens and an insect. Am Phytopathol Soc 71, 297-301
Massonié G (1986) Influence des substances secondaires des plantes sur le comportement alimentaire des pucerons. In : La nutrition des crustacées et des insectes (CNERMA, ed), 293-303

Massonié G, Maison P (1979) Résistance de 2 variétés de Prunus persica (L) Batsch à Myzus persicae Sulzer et à Myzus varians Davidson : étude préliminaire des mécanismes de résistance. Ann Zool Ecol Anim 11, 479-485

Massonié G, Maison P, Monet R, Grassely L (1982) Résistance au puceron vert du pêcher Myzus persicae Sulzer chez Prunus persica (L) Batsch et d'autres espèces de Prunus, agronomie 2, 73-70

Miles PW (1989) Specific responses and damage caused by Aphidoidea. In : Aphids. Their Biology, Natural Enemies and Control (AK Minks, P Harrewijn, eds), Elsevier, Amsterdam, 2c, 23-42

Miles PW (1990) Aphid salivary secretions and their involvement in plant toxicoses. In : Aphid-Plant genotype Interactions (RK Campbell, RD Eikenbary, eds), Elsevier, Amsterdam, 131-147

Niemeyer HM, Pesel E, Copaja JV, Bravo HR, Franke $S$, Francke W (1989) Changes in hydroxamic acid levels of wheat plants inducd by aphid feeding. Phytochem 28, 2, 447-449

Ryals J, Uknes S, Ward E (1994) Systemic acquired resistance. Plant Physiol 104, 1109-112

Scherrer B (1984) Biostatistique. G Morin, $850 \mathrm{p}$

Van der Westhuizen AJ, Botha FC (1993) Effect of the Russian wheat aphid on the composition and synthesis of water soluble proteins in resistant and susceptible wheat. J Agron Crop Science 170, 322-326

Way MJ, Cammell M (1970) Aggregation behaviour in relation to food utilization by aphids. In : Animal Populations in their Relations to Food Resources (A Watson, ed), Blackwell Scientific, Oxford, 229-247

Westphal E, Dreger F, Bronner R (1991) Induced resistance in Solanum dulcamara triggered by the gall mite Aceria cladophtirus (Acari: Eriophyoidea). Exp Applied Acarol 12, 111-118

Westphal E, Perrot-Minnot MJ, Kreiter S, Gutierrez J (1992) Hypersensitive reaction of Solanum dulcamara to the gall mite Aceria cladophtirus causes an increased susceptibility to Tetranychus urticae. Exp Applied Acarol 15, 15-26 